\title{
FLAT MICROWAVE SPECTRA SEEN AT X-CLASS FLARES
}

\author{
JEONGWOO W. LEE, DALE E. GARY and H. ZIRIN \\ California Institute of Technology, Pasadena, CA 91125, U.S.A.
}

(Received 27 October, 1993; in revised form 28 February, 1994)

\begin{abstract}
We report peculiar spectral activity of four large microwave bursts as obtained from the Solar Arrays at the Owens Valley Radio Observatory during observations of X-class flares on 1990 May 24 and 1991 March 7,8, and 22. Main observational points that we newly uncovered are: (1) flat flux spectra over $1-18 \mathrm{GHz}$ in large amounts of flux ranging from $10^{2}$ to $10^{4}$ s.f.u. at the maximum phase, (2) a common evolutionary pattern in which the spectral region of dominant flux shifts from high frequencies at the initial rise to low frequencies at the decaying phase, and (3) unusual time profiles that are impulsive at high frequencies but more extended at lower frequencies.

In an attempt to elucidate these new properties, we carry out the model calculations of microwave spectra under assumptions of gyrosynchrotron mechanism and a dipole field configuration to reproduce the observational characteristics. Our results are summarized as follows. First, a flat microwave spectrum reaching up to $10^{2}-10^{4}$ s.f.u. may occur in a case where a magnetic loop is extended to an angular size of $\sim(0.7-7.0) \times 10^{-7}$ sterad and contains a huge number $\left(N(E>10 \mathrm{keV}) \sim 10^{36}\right.$ $10^{38}$ ) of nonthermal electrons with power-law index $\delta \sim 3-3.5$ over the entire volume. Second, the observed spectral activity could adequately be accounted for by the shrinking of the region of nonthermal electrons to the loop top and by the softening of the power-law spectrum of electrons in a time scale ranging 3-45 min depending on the event. Third, the extended microwave activity at lower frequencies is probably due to electrons trapped in the loop top where magnetic fields are low. Finally, we clarify the physical distinction between these large, extended microwave bursts and the gradual/post-microwave bursts often seen in weak events, both of which are characterized by long-period activity and broadband spectra.
\end{abstract}

\section{Introduction}

The large microwave bursts which are characterized by high fluxes over $10^{3}$ solar flux units (s.f.u.) and a flat spectral shape over 1-20 GHz have been reported by Hachenberg and Wallis (1961). Most attention was given to the flat spectral shape, which is against the prediction of the theory of gyrosynchrotron radiation from a uniform source (cf., Ramaty and Petrosian, 1972; Klein and Trottet, 1984). Since no further reports of such flat and high microwave flux spectra followed after Hachenberg and Wallis (1961), the theoretical efforts have been limited to those addressing only the instantaneous spectral shape at the maximum phase (Ramaty and Petrosian, 1972; Klein and Trottet, 1984). For the purpose of further exploring properties of this kind of microwave activity, we examined the data gathered from the solar-dedicated telescope at the Owens Valley Radio Observatory (OVRO) over a few years around the last solar maximum to find four samples, the 1990 May 24 and 1991 March 7, 8, and 22 flares, which show a similar spectral behavior at the maximum phase. Having examined these data in time as well as with high spectral resolution, we find the following properties are worthy of new attention.

First, these microwave bursts exhibit radio fluxes ranging from $10^{2}$ to $10^{4}$ s.f.u. The latter value is comparable to the highest microwave fluxes ever reported. As 
examples, Croom and Powell (1971) reported two events with fluxes over $10^{4}$ s.f.u. at $19 \mathrm{GHz}$ among 148 bursts gathered over 2.5 years; Tanaka (1969) stated that the highest microwave flux ever recorded at $3 \mathrm{GHz}$ was $3.2 \times 10^{4}$ s.f.u. in the 1969 March 30 flare; the 1991 June 6 flare attained $\sim 5 \times 10^{4}$ s.f.u. at $2.7 \mathrm{GHz}$ (Solar Geophysical Data, 1991, Prompt Reports, No. 564). Explanation of such a high level of flux may require a new quantitative model simply because it far exceeds the previously known maximum flux value used as an observational constraint in a theoretical model (Ramaty and Petrosian, 1972). Second, temporal evolution of these burst spectra appears in a common pattern characterized by dominant flux at high frequencies at the initial rise, flat shape at the maximum phase, and dominant flux at low frequencies at the decay phase. Such an evolutionary pattern might be indicative of a common dynamical origin for these events. Third, the microwave decay time, in these samples, shows a large variation with frequency as follows: the higher frequency fluxes are as impulsive as hard X-rays, while lower frequency fluxes are as extended as soft X-rays. This provides a challenge to the well-known rule that the time profile of microwaves in general resembles that of hard X-rays and its time-integral reproduces that of soft X-rays (e.g., Neupert, 1968; Tanaka and Zirin, 1985; Dennis and Zarro, 1993).

These new observational properties therefore pose additional problems that must be addressed by the models which were originally constructed to account for the flat spectral shape at the maximum phase. In particular, the fact that this distinctive spectral activity occurs in several large events suggests that the origin of such anomalies should be looked for in the characteristics of big flares. As an effort in this line, we carry out model-calculations of microwave flux under assumptions of nonthermal gyrosynchrotron mechanism and dipole field geometry mainly to deduce source conditions for the observed spectral properties during the flares. We also show that the results of spectral fitting can lead to a qualitative understanding of the unusual frequency-dependent relationship between the microwaves and Xrays in these events. In this way, we hopefully explore the big flare characteristics underlying these large microwave bursts with peculiar spectral properties. The plan of this paper is as follows: in Section 2 we describe the observations. In Section 3 we set up our line of approach by a review of previous works that may bear on the explanation of the present observations. In Section 4, our model calculations are presented and the issues are discussed based on the results. Conclusions are given in Section 5.

\section{Observation}

Data we examined are microwave spectra and soft X-ray time profiles of four Xclass flares that occurred on 1990 May 24 and 1991 March 7, 8, and 22. Microwave spectra are obtained from the OVRO Solar Array with spectral resolution of $0.2 \mathrm{GHz}$ in the frequency range of $1-18 \mathrm{GHz}$ and time resolution of $10 \mathrm{~s}$. The soft X-ray fluxes at 1-8 $\AA$ are obtained from the GOES satellite. The flaring time, 
TABLE I

Observational data

\begin{tabular}{rlllrll}
\hline Date & Region & Location & Peak time & Duration & Maximum flux & $\begin{array}{l}\text { SX- } \\
\text { class }\end{array}$ \\
\hline 24 May, 1990 & AR 6063 & N31 W66 & $20: 48: 07$ & $\sim 40 \mathrm{~min}$ & $1.4 \times 10^{4}$ s.f.u. & X9.3 \\
7 Mar., 1990 & AR 6538 & S21 E67 & $23: 17: 46$ & $9.5 \mathrm{~min}$ & $1.7 \times 10^{2}$ s.f.u. & X2.5 \\
8 Mar., 1990 & AR 6538 & S25 E50 & $20: 27: 16$ & $36 \mathrm{~min}$ & $2.0 \times 10^{3}$ s.f.u. & X1.7 \\
22 Mar., 1990 & AR 6555 & S27 E27 & $22: 44: 12$ & $>80 \mathrm{~min}$ & $1.4 \times 10^{4}$ s.f.u. & X9.4 \\
\hline
\end{tabular}

source location, and maximum fluxes of microwaves and soft X-ray classes of these events are listed in Table I. It is noted that the four events are all characterized by high flux level at the maximum phase $\left(10^{2}-10^{4}\right.$ s.f.u. $)$ and long durations of the whole activity (10 min to hours). We describe the microwave spectra of these events in Section 2.1 and their time correlations with X-rays in Section 2.2.

\subsection{MICROWAVE SPECTRA AND THEIR TIME-EVOLUTION}

Figure 1 shows the time-sequence of total power spectra centered at the time of maximum flux of four events. At the maximum phase (the central frames in Figure 1), the spectra appear in a broadband type (Guidice and Castelli, 1975) and similar to those reported by Hachenberg and Wallis (1961). It is noteworthy that two of them show fluxes above $10^{4}$ s.f.u., which even persist to frequencies as low as $1 \mathrm{GHz}$. It is one of the largest radio fluxes ever reported at that low frequency (see Section 1).

Another interesting point in Figure 1 is the characteristic pattern of the spectral evolution which takes three stages. In the initial impulsive rise phase, a spectral peak shows up first at high frequencies and then subsequent enhancement of flux follows up at lower frequencies (the first and the second frames in Figure 1). In the maximum phase, the overall spectra appear in a flat shape (the central frame). In the decay phase, the higher frequency components diminish much faster while lower frequency ones diminish more slowly. As a result, fluxes dominate at low frequencies with negative spectral slope in the extended decay phase and the negative slope is gradually increasing with time (the fourth and the last frames).

Such a change of spectral shape during flares is in a contrast with a more common case of impulsive microwave bursts where the spectra evolve keeping the same shape during flares (Stähli, Gary, and Hurford, 1989, 1990).

\subsection{TIME PROFILES OF MICROWAVES AND X-RAYS}

In Figure 2 we plot the microwave fluxes at four frequencies $(1,2,5$, and $18 \overrightarrow{\mathrm{GHz}})$ in upper panels and X-ray fluxes at 1-8 $\AA$ in lower panels. As hard X-ray data from HXRBS were unavailable at the times of these events, we plot the time- 


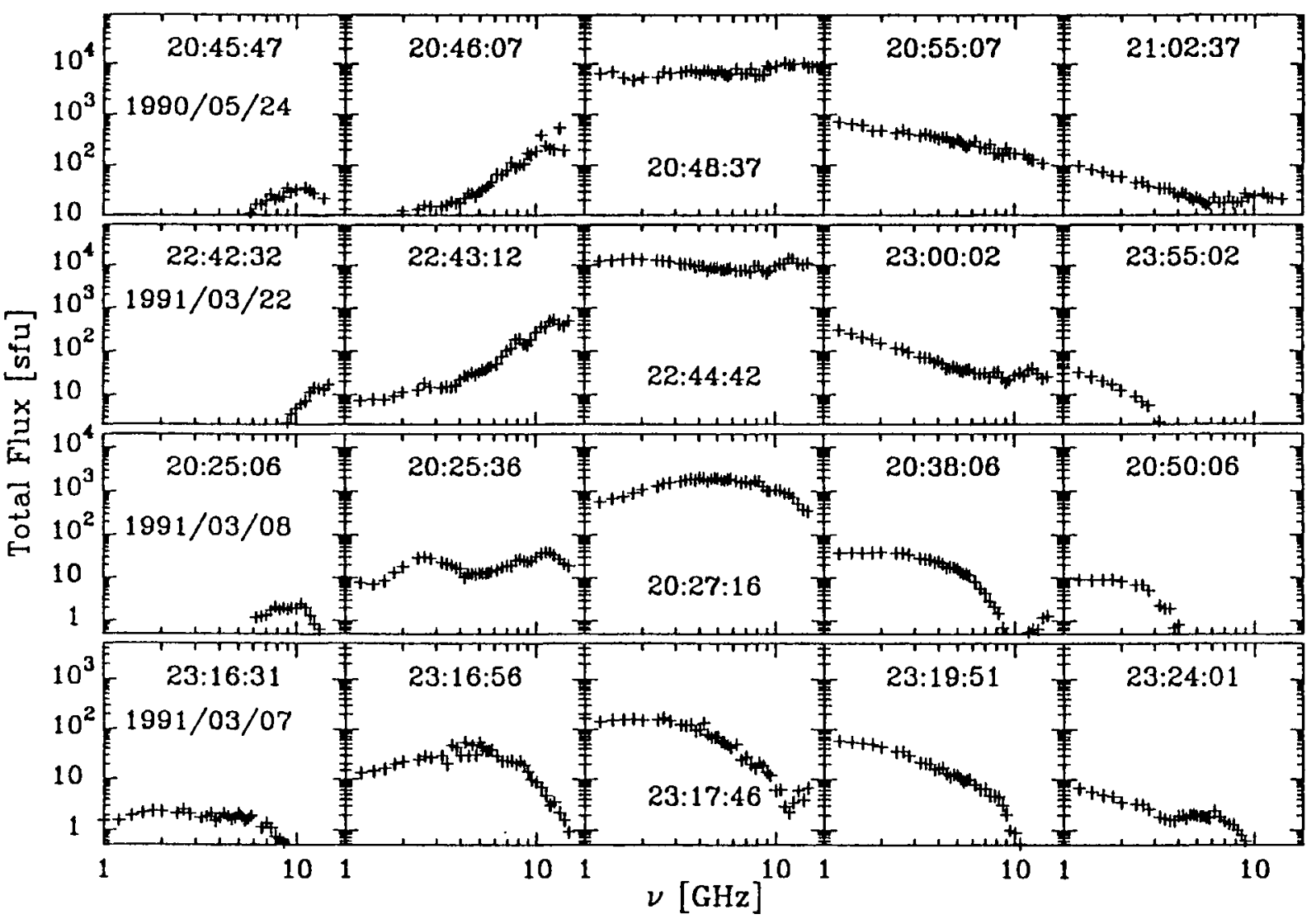

Fig. 1. Time-sequence of the observed total power spectra in four events as listed in Table I. The frames in each event are centered on the time of maximum phase.

derivative of the soft X-ray curve as a guide line for expected hard X-ray curve in light of the well-known 'Neupert effect' (Neupert, 1968; Dennis and Zarro, 1993). The hypothetical hard X-ray curves computed in this way show duration over 1-2 min (dotted lines in Figure 2). These values are in a rough agreement with the data of the Phebus experiment on board the GRANAT satellite in high-energy range $(0.42-0.60 \mathrm{MeV})$ for the 1991 March 22 event (C. Barat and O. Terekhov, 1993, private communication). The 1990 May 24 event has been observed in wide energy range. The Phebus data of this event shows that the burst in the energy range 0.84-1.1 MeV lasts over 1-2 min similar to the above case (Talon et al., 1993); the Sigma data shows the burst in 41-163 keV, however, lasts over $10 \mathrm{~min}$ (Pelaez et al., 1992).

GOES soft X-ray data are usually given in two wavelength bands to provide information of the electron temperature, $T_{e}$, and the emission measure, $\int n_{e}^{2} \mathrm{~d} V$ (Thomas, Starr, and Crannell, 1985). In the present data, the fluxes at wavelengths 0.5-4 $\AA$ show saturation near the peak time. If we make interpolation to recover the saturated portion approximately, a rough estimate of these quantities goes as $\int n_{e}^{2} \mathrm{~d} V \approx 9 \times 10^{50} \mathrm{~cm}^{-3}$ for the 1990 May 24 event, $8 \times 10^{50} \mathrm{~cm}^{-3}$ for the 1991 March 22 event, and $2 \times 10^{50} \mathrm{~cm}^{-3}$ for the 1991 March 7 event and $T_{e} \approx 2 \times 10^{7} \mathrm{~K}$ for all these three events; GOES data for the 1991 March 8 event are absent. These 


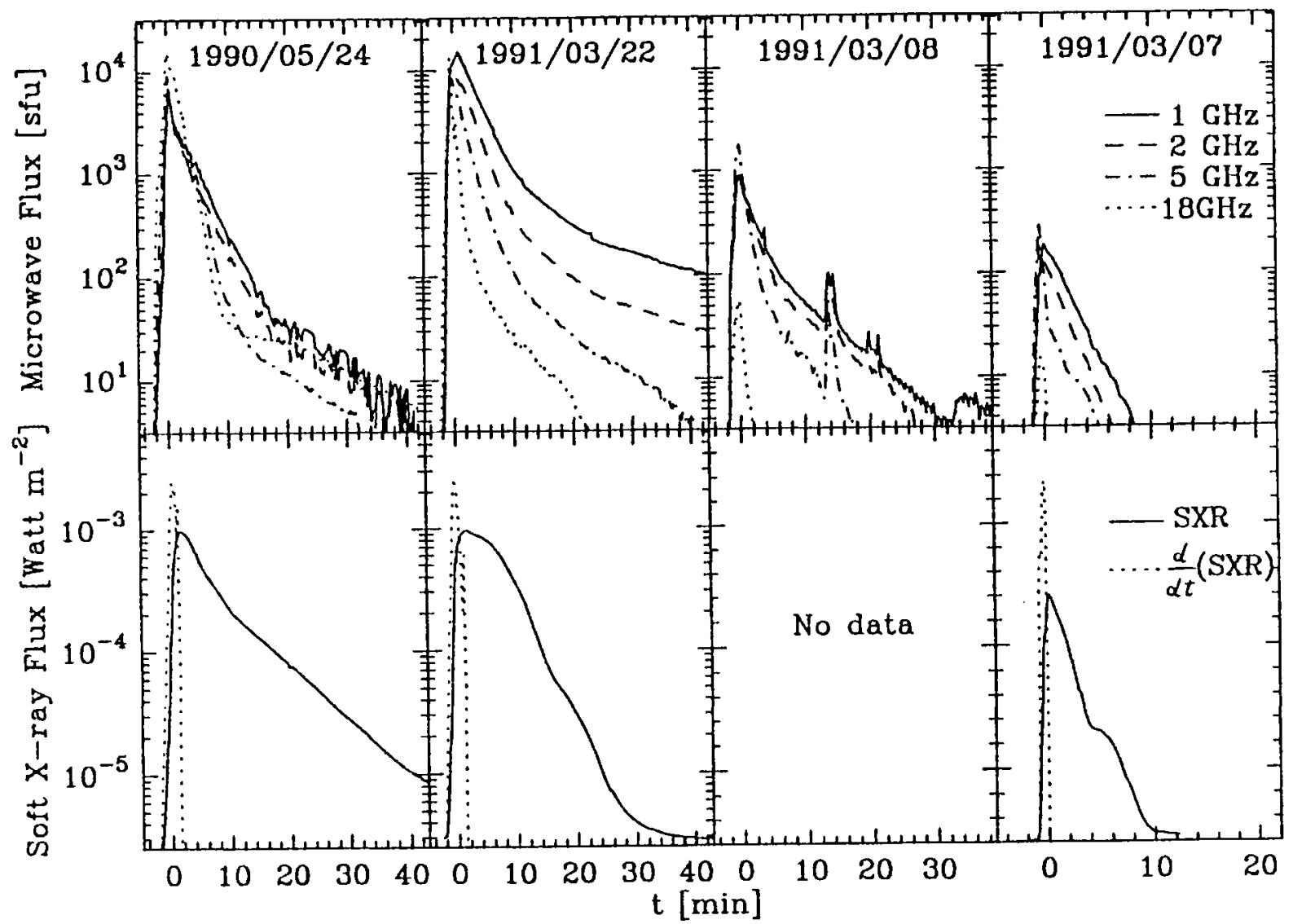

Fig. 2. Comparison of microwave time profile with X-ray light curves. Upper panels: microwaves at four selected frequencies in 1-18 GHz. Lower panels: soft X-ray light curves at $1-8 \AA$ from GOES (solid lines) and their time-derivatives taken as hypothetical hard X-ray curves (dotted lines). $t=0$ in $x$-axes correspond to the peak times of the events which are 20:48:07 UT (1990 May 24), 22:44:12 UT (1991 March 22), 20:27:16 UT (1991 March 8), and 23:17:46 UT (1991 March 7), respectively.

results suggest that the events under present investigation belong to the strongest class of soft X-ray activity (cf., Denton and Feldman, 1984).

Comparison of microwave time profiles with those of X-rays yields several points to note. First, the well-known good correlation between microwaves and hard X-rays (e.g., Dennis, 1988) is found only at high frequencies ( $>14 \mathrm{GHz}$ ). Second, at lower frequencies $(<14 \mathrm{GHz}$ ), the microwave flux violates this rule by decaying more slowly. This trend of longer duration at lower frequencies continues down to $1 \mathrm{GHz}$, the lowest frequency used in the present observation, so that the microwaves at $1 \mathrm{GHz}$ approach closer to that of soft X-rays. Third, the degree of closeness of microwaves at $1 \mathrm{GHz}$ with soft $\mathrm{X}$-rays is, however, different from event to event. In particular, microwaves at all frequencies decay faster than soft $X$ rays in the 1990 May 24 event while microwaves below $5 \mathrm{GHz}$ live longer than soft $\mathrm{X}$-rays in the 1991 March 22 event. We also note that microwaves rise impulsively at all frequencies while soft X-rays rise more slowly and peak later as compared to the microwaves in all events. Due to such discrepancies, it is unlikely that the microwaves at lower frequencies share the same origin with soft $\mathrm{X}$-rays although 
duration times are roughly similar. The general trend for increasing microwave duration toward lower frequencies must, however, be explained.

\section{Previous Studies}

The above observational properties may be new as a whole. However, the flat spectra and the long-duration activity seen at large bursts have been discussed elsewhere referring to different observations. For the purpose of gathering available information, we review the previous studies here and discuss their relevance to understanding of the present data.

\subsection{ON THE FLAT SPECTRAL SHAPE}

It were Ramaty and Petrosian (1972) who first gave a theoretical interpretation of flat and high microwave flux spectra as observed by Hachenberg and Wallis (1961), in terms of a uniform model. They noted that the free-free emission is not capable of producing the high microwave fluxes as observed $\left(\sim 10^{3}\right.$ s.f.u.) and that nonthermal gyrosynchrotron emission is thus the most likely radiation mechanism. In a uniform model, however, the flat spectral shape is hardly reproduced by the gyrosynchrotron mechanism alone so that they worked out a set of conditions for dominance of the free-free opacity relative to the gyrosynchrotron opacity over 2$20 \mathrm{GHz}$. The resulting conditions include low electron temperature, $T_{e}<3 \times 10^{6} \mathrm{~K}$, and weak magnetic field, $B<180 \mathrm{G}$, and a limit for the possible maximum flux as $F_{\nu} \leq 2 \times 10^{3}$ s.f.u.

Klein and Trottet (1984), on the other hand, proposed that source inhomogeneity can lead to flattening of microwave spectra due to nonthermal gyrosynchrotron. Klein, Trottet, and Magun (1986) noted that multiple burst sources can lead to a similar effect. The flattening of optically-thick nonthermal gyrosynchrotron spectra due to source inhomogeneity was noticed earlier by Takakura and Scalise (1970) and Takakura (1972) although no particular observation was referenced. Böhme et al. (1977) suggested superposition of multiple sources to produce broadband spectra. In a somewhat different context, Dulk and Dennis (1982) also noted that multi-thermal components will lead to flattening of optically-thick thermal gyrosynchrotron flux spectra.

In our view, a nonuniform model comes closer to explaining the present observation than the uniform model by Ramaty and Petrosian (1972). First of all, in the uniform model, any attempt to raise the flux up to the observed high values using gyrosynchrotron emission is subject to the restriction that free-free opacity must dominate, which limits the brightness temperature. A nonuniform model has no such restriction. Second, according to Ramaty and Petrosian's (1972) results, a large source area above $\sim 10^{-7}$ sterad is likely to be needed to explain the high flux above $10^{3}$ s.f.u. at low frequencies. Extending source area above that large value, still keeping a uniform structure, seems unrealistic in view of the typical physical scale of solar active regions. It is likely that such a big source is inevitably 
inhomogeneous and the flat spectra are a natural consequence of the source inhomogeneity.

\subsection{ON THE LONG-DURATION ACTIVITIES}

Another characteristic of our data is the long duration at low frequencies. This recalls several works discussing the underlying physics of extended bursts in contrast to that of impulsive bursts, based on time profiles of microwaves and hard X-rays (Vilmer, Kane, and Trottet, 1982; Kai, Kosugi, and Nitta, 1985; Kosugi, Dennis, and Kai, 1988; Bruggmann et al., 1994). A basic notion brought up by these papers is that a coronal trapping of energetic particles is responsible for the long duration microwave activity in general. This coronal trap model appears to be useful in explaining the extended microwave activity at lower frequencies if it is combined with the nonuniform magnetic field model. Namely, lower frequency microwaves are emitted from weaker field region near the loop top where particles are trapped and thus survive longer. The high-frequency microwaves are still expected to be short-lived as they are emitted from a stronger field region near footpoints. With a uniform loop model, we hardly make such a distinction of microwave duration with frequency. That is another benefit of a nonuniform model.

\section{Analysis with Model Support}

In light of the foregoing discussion, we analyse the observed microwave spectra using a nonuniform magnetic loop model and gyrosynchrotron radiation mechanism. The model is described in Section 4.1. Using this model, we interpret and analyse the flat and high flux spectra observed at the maximum phase in Section 4.2. The time-evolution of microwave spectra is analysed in Section 4.3. The result obtained in Section 4.3 is used again to give a qualitative interpretation of the correlations of microwaves with X-rays in Section 4.4.

\subsection{MODEL}

In the absence of spatial information of the radio-emitting region, we tentatively adopt a magnetic loop model as shown in Figure 3. The magnetic fields inside of the loop are assumed to be due to a single dipole lying parallel to the photosphere as in Klein and Trottet's (1984) model (cf., Takakura and Scalise, 1970; Alissandrakis and Prekka-Papadema, 1984; Prekka-Papadema and Alissandrakis, 1988, 1992; Kucera et al., 1993, for the other model set-up). Size of the loop is parametrized by the height $R$ and the thickness at the apex, $2 \Delta R$. The coronal field distribution is scaled according to $B_{1}$ and $B_{2}$ which are the field strength at the middle of the loop apex and that at the coronal base, respectively (see Figure 3). We set $B_{2}=1500 \mathrm{G}$ in this study.

The model dipole loop set up in the reference frame $\left(x_{0}, y_{0}, z_{0}\right)$ is rotated by heliographic angles $(\theta, \phi)$ so that the distribution of field strengths and viewing 


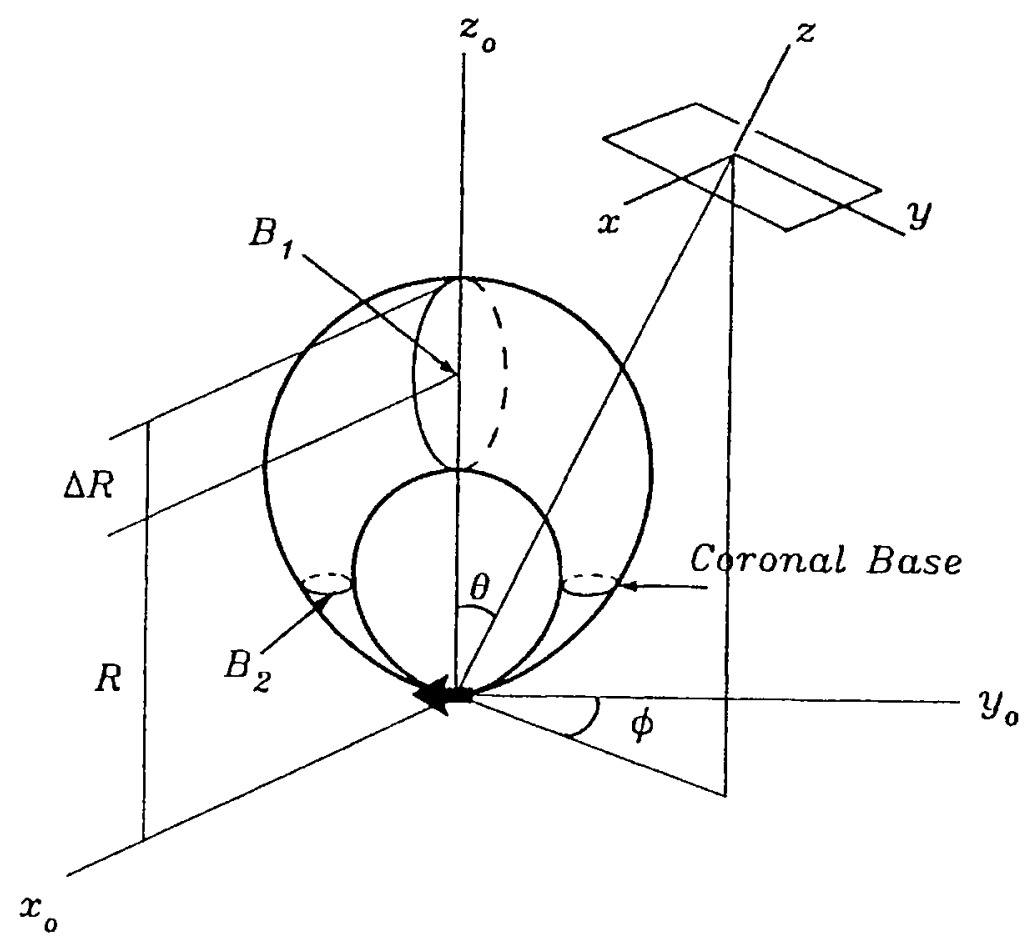

Fig. 3. A model configuration of a dipole loop as seen by an observer at $(\theta, \phi) .\left(x_{0}, y_{0}, z_{0}\right)$ is the reference frame of the loop and $(x, y, z)$ is the observer's frame with $\hat{z}$ along the line of sight. $R$ and $\Delta R$ are the height and the thickness of the loop at the apex, respectively; $B_{1}$ and $B_{2}$ represent the field strength at the middle of loop apex and that at the coronal base, respectively.

angles can be determined in the observer's frame $(x, y, z)$. At each point $(x, y z)$ inside the loop, we calculate the emissivity $j_{\nu}$ and the opacity $\tau_{\nu}$ due to gyrosynchrotron radiation and perform the line-of-sight ( $\hat{z}$ in Figure 3 ) integration of the radiative transfer equation to get the distribution of the brightness temperature, $T_{B}$, on the projected sky plane $(x, y)$ as

$$
T_{B, \nu}(x, y)=\frac{c^{2}}{k \nu^{2}} \int_{0}^{\theta} j_{\nu} e^{-\tau_{\nu}} \mathrm{d} z,
$$

where $k$ is the Boltzmann constant and $c$ is the speed of light. To calculate $j_{\nu}$ and $\tau_{\nu}$, we use the simplified expressions by Dulk and Marsh (1982) mainly for tractability of the calculation with our three-dimensional loop model (cf. Klein and Trottet, 1984; Preka-Papadema and Alissandrakis, 1992). Dulk and Marsh's (1982) approximation is, however, made valid over a moderate range of harmonics $\left(10 \leq \nu / \nu_{B} \leq\right.$ a few hundred). We will discuss the possible uncertainty at the very low and high harmonics in Section 5 together with our results. From the brightness distribution, $T_{B, \nu}(x, y)$, we calculate effective source area and total flux,

$$
\Omega_{\mathrm{eff}}(\nu)=\frac{\int T_{B, \nu}(x, y) \mathrm{d} x \mathrm{~d} y}{T_{B, \nu}^{\max }} \quad \text { and } \quad F_{\nu}=k T_{B, \nu}^{\max }\left(\frac{\nu}{c}\right)^{2} \Omega_{\mathrm{eff}}(\nu)
$$


as a function of frequency where $T_{B, \nu}$ is the maximum value of $T_{B, \nu}^{\max }(x, y)$.

In the present model computation, we mainly control (i) the size of the loop, $R$ and $\Delta R$, and the field strength at the loop top, $B_{1}$, all of which determine the degree of magnetic field inhomogeneity and (ii) the power-law index of electrons, $\delta$, which defines hardness of the electron energy distribution. For simplicity, we assume that the nonthermal electrons are uniformly distributed inside the loop.

\subsection{FLAT SPECTRAL SHAPE AND MAXIMUM FLUX}

The goal here is to investigate under which source condition the observed flat and high-flux spectra are reproduced. As a first step, we check the issue of ther$\mathrm{mal} /$ nonthermal origin of these events and also the uniform/nonuniform sources. We show the results in Figure 4. The upper panels in Figure 4 show two-dimensional display of $T_{B, \nu}$ for nonthermal (Figure 4(a)) and thermal (Figure 4(b)) gyrosynchrotron emission from a nonuniform magnetic loop and the nonthermal gyrosynchrotron from a uniform loop (Figure 4(c)) mainly to contrast the source areas at two different frequencies. In the lower panel we show the spectra of maximum brightness temperatures (Figure 4(d)), effective source area (Figure 4(e)), and the total flux (Figure 4(f)); model parameters used in the calculation are listed below the figure. It is known from previous works that under certain circumstances the whole loop is bright at lower frequencies while only the footpoints are bright at high frequencies (e.g., Klein and Trottet, 1984; Alissandrakis and Prekka-Papadema, 1984; Alissandrakis, 1986; Prekka-Papadema and Alissandrakis, 1988, 1992). This tendency is particularly prominent in the present models (Figures 4(a) and 4(b)) where the field strengths at the loop top and footpoints make a big contrast $\left(B_{1} / B_{2} \approx \frac{1}{300}\right)$. The uniform model (Figure 4(c)), however, does not share this property so that $\Omega_{\text {eff }}$ changes little with frequency (Figure 4(e)). As a result, total flux spectrum follows closely the Rayleigh-Jeans law: $F_{\nu} \sim \nu^{2}$ (Figure 4(f)). In nonuniform models, the effective source size increases towards lower frequencies (Figure 4(e)) and it tends to make the resulting flux spectra flatter than the $\nu^{2}$-slope (Figure 4(f)).

With the frquency-dependence of source size alone, however, an essentially flat flux spectrum cannot be achieved; we thus need an increase of $T_{B}(\nu)$ towards lower frequencies as well. Figure 4(d) shows that the increase of $T_{B}(\nu)$ at lower frequencies can happen for nonthermal gyrosynchrotron radiation. To understand the increase of $T_{B}(\nu)$ at lower frequencies, we recall that the fact that for nonthermal particles, the effective temperature depends on magnetic fields and power-law index as $T_{\text {eff }} \equiv\left(c^{2} / k \nu^{2}\right)\left(\eta_{\nu} / \kappa_{\nu}\right) \sim\left(\nu / \nu_{B}\right)^{0.5+0.085 \delta}$ (Dulk and Marsh, 1982) where $\nu_{B}$ (三2.8B MHz) is the gyrofrequency. Therefore, $T_{\text {eff }}$, which corresponds to the optically thick brightness temperature, can be made higher towards lower frequencies if the effective source region moves to weaker field regions at lower frequencies to increase $\left(\nu / \nu_{B}\right)$. Figure 4(a) shows that this is the case for nonthermal gyrosynchrotron emission from a nonuniform magnetic loop. In casè of a thermal model, $T_{\text {eff }}$ is equivalent to the electron temperature, $T_{e}$, independent of frequency and therefore a thermal model is inadequate in producing the flat and 

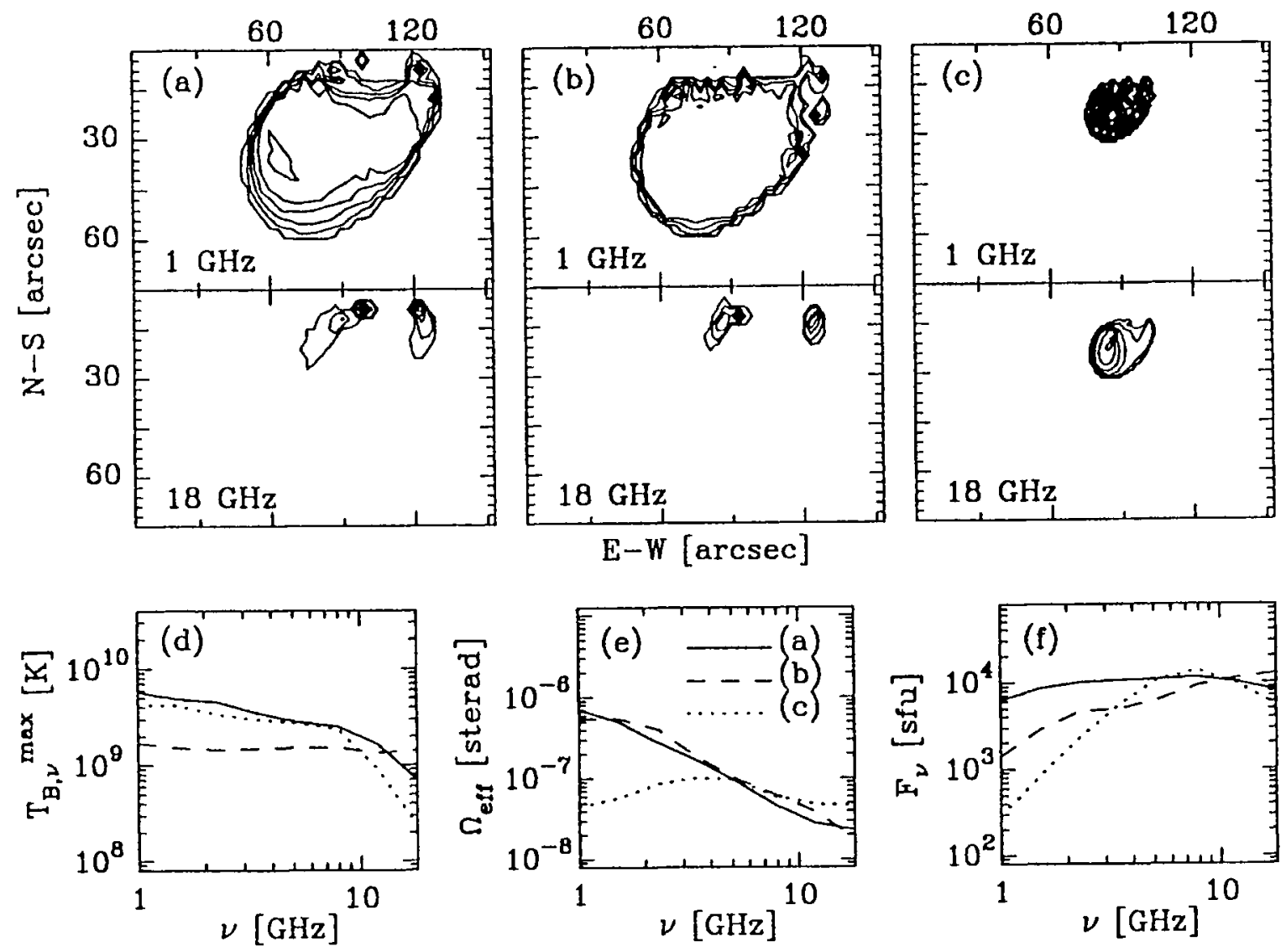

Fig. 4. Upper panels: model-calculation of the brightness temperature distribution for (a) nonthermal gyrosynchrotron radiation, (b) thermal gyrosynchrotron radiation from a nonuniform source, and (c) nonthermal gyrosynchrotron radiation from a uniform source. Lower panels: calculated spectra of (d) the brightness temperature, (e) effective source area, and (f) total flux for the above three cases: case (a) solid lines, case (b) dashed lines, and case(c) dotted lines.

high-flux spectra as we observed. To conclude, only nonthermal gyrosynchrotron emission from an inhomogeneous source can explain the spectral characteristics we observe.

We now examine the influence of various source parameters upon the resulting flux spectra using only the nonthermal gyrosynchrotron source. Figure 5(a) shows flux spectra calculated with three different values of field strength at the loop top, $B_{1}$; flux spectra become flatter as $B_{1}$ decreases and thus makes bigger contrast with the field strength at the base of the loop. Figure 5(b) (bottom panel) shows flux variation with loop size; in order to raise the amount of flux up to $10^{4}$ s.f.u., we need a gigantic loop with $R \approx 300^{\prime \prime}$, which will then result in a radio source of size $\sim 7 \times 10^{-7}$ sterad at $1 \mathrm{GHz}$. We note that a large microwave burst with a size comparable to the above values has been reported by Kucera et al. (1994). Results shown in Figures 5(a) and 5(b) give an estimate for the field strength and source size required to reproduce the observed flux spectra (Figure 1). Figure 5(c) shows dependence of spectra on the loop location on the solar disk. For a fixed heliolatitude $\left(\phi=25^{\circ}\right)$, we try three heliolongitudes, $\theta=0^{\circ}, 30^{\circ}$, and $70^{\circ}$, to find that the resulting spectra are insensitive to heliographic location. This result 


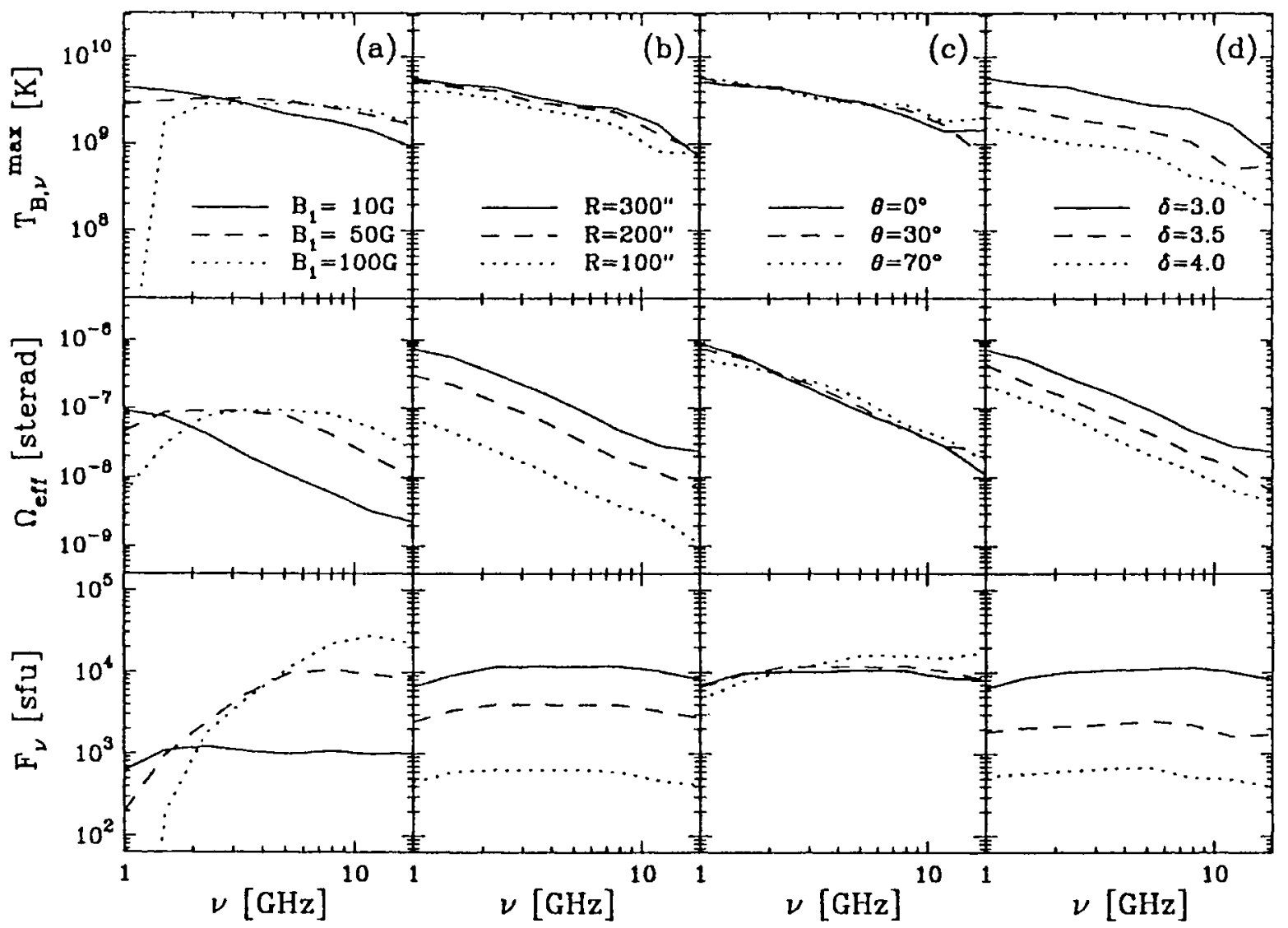

Fig. 5. Calculated spectra of the brightness temperature, source area, and total flux: their variations with (a) magnetic field strength at the loop top, $B_{1}$, (b) loop size, $R$, (c) heliolongitude, $\theta$, and (d) power-law index, $\delta$, of nonthermal electrons.

disagrees with previous results (e.g., Takakura and Scalise, 1970) which show a limb increase of gyrosynchrotron flux. We note that such a directivity results when the particles are assumed to be concentrated at a lower coronal region where the field strength is relatively high and the field directions are better organized. This is not the case in our model, where the particles are distributed over the whole loop which is so highly inflated that the field distribution as a whole loses directivity. Our result of the insensitive dependence of flux shape upon the heliographic position is in agreement with the observation (see Table I for the source location). Finally, we check the dependence of flux on the hardness of radiating particles in Figure 5(d). The result shows that a relatively hard electron distribution $(\delta \approx 3)$ is essential to produce the high level of flux as boserved $\left(F_{\nu} \approx 10^{4}\right.$ s.f.u. $)$ in addition to the big size $\left(R \approx 300^{\prime \prime}\right)$ and a large degree of source inhomogeneity $\left(B_{1} / B_{2} \approx \frac{1}{300}\right)$.

Based on the results presented in Figure 5, we determine sets of source parameters that best reproduce the observed spectra at the maximum phase (Figure 1); results are listed in Table II (columns 2-6). Taking a weaker field strength at the loop top $(5-10 \mathrm{G})$ is essential to reproduce the flat flux spectra as observed in all events. Hard nonthermal electrons $(\delta=3)$ and a large source size $\left(R=300^{\prime \prime}\right)$ are required to raise the overall fluxes to those of two strongest events (the 1990 
TABLE II

Derived model parameters

\begin{tabular}{lclllll}
\hline \multicolumn{1}{l}{ Event } & $B_{1}(\mathrm{G})$ & $R\left(^{\prime \prime}\right)$ & $\Omega_{1} \mathrm{GHz}$ (sterad) & $N(E \geq 10 \mathrm{keV})$ & $\delta$ & $\tau_{\delta}(\mathrm{min})$ \\
\hline 24 May, 1990 & 5 & 300 & $7 \times 10^{-7}$ & $3 \times 10^{38}$ & 3.0 & $\geq 40$ \\
7 Mar., 1991 & 10 & 180 & $7 \times 10^{-8}$ & $7 \times 10^{35}$ & 3.5 & $\sim 3.3$ \\
8 Mar., 1991 & 10 & 240 & $3 \times 10^{-7}$ & $1 \times 10^{37}$ & 3.5 & $\sim 6.8$ \\
22 Mar., 1991 & 5 & 300 & $7 \times 10^{-7}$ & $3 \times 10^{38}$ & 3.0 & $\sim 46$ \\
\hline
\end{tabular}

May 24 and 1991 inarch 22 flares). For weaker events (the 1991 March 8 and 1991 March 7 flares), relatively softer electrons $(\delta=3.5)$ and smaller source sizes $\left(R=180^{\prime \prime}-240^{\prime \prime}\right)$ are adequate. Total number of nonthermal electrons above $10 \mathrm{keV}$ is calculated for each event by integrating the electron density over the whole loop volume. The resulting values, $N(E \geq 10 \mathrm{keV}) \approx 10^{36}-10^{38}$, far exceed commonly reported values of normal flares but are comparable to the previous result by Ramaty and Petrosian (1972) for the flat spectra observed by Hachenberg and Wallis (1961), which are similar to that of the 1991 March 8 event.

\subsection{TIME-EVOLUTION OF MICROWAVE FLUX SPECTRA}

Another interesting property of these microwave bursts is the time-evolution of the microwave spectra. In the present study, we investigate a hypothesis that a timedependent change of source position within a nonuniform magnetic loop can lead to such a time behavior, in light of the sensitive dependence of gyrosynchrotron spectra on magnetic fields. We here describe our procedure mainly referring to the spectra from the 1991 March 22 flare. In Figure 6, we make a model-fit to the observed spectra at five instances: $t_{1}$ (22:43:20 UT), $t_{2}$ (22:43:50 UT), $t_{3}$ (22:44:10 UT), $t_{4}(22: 55: 00 \mathrm{UT})$, and $t_{5}(23: 30: 00 \mathrm{UT})$ in the 1991 March 22 event.

We first consider the spectra in the rise phase of the 1991 March 22 event. The spectra at this period show flux concentrated at high frequencies, implying that radiation comes from a relatively stronger and more uniform field region. Since there is no good reason that nonthermal electrons should be confined only to a stronger field region in our loop geometry, such a spectrum is hardly reproduced if we keep the same source size $R$ and the same field strength $B_{1}$ as we assumed to match the maximum phase spectra. As a plausible model, we assume that the loop was initially of a smaller size, but expands to a size of $R \approx 300^{\prime \prime}$ at the maximum phase. We thus make a fit to the observation by using $R=40^{\prime \prime}$ and $\Delta R / R=0.1$ at $t_{1}$ and $R=200^{\prime \prime}, \Delta R / R=0.2$ at $t_{2}$; the magnetic field strengths scale according to loop height $R$ as $B_{1}\left(t_{1,2}\right) \approx B_{1}\left(t_{3}\right)\left[R\left(t_{3}\right) / R\left(t_{1,2}\right)\right]^{2}$ (Figure 6(a)). On the other hand, the change of $\delta$ has little influence on the shape of spectra which are optically thick at the frequencies under investigation. Therefore, it is most likely that the 
(a)

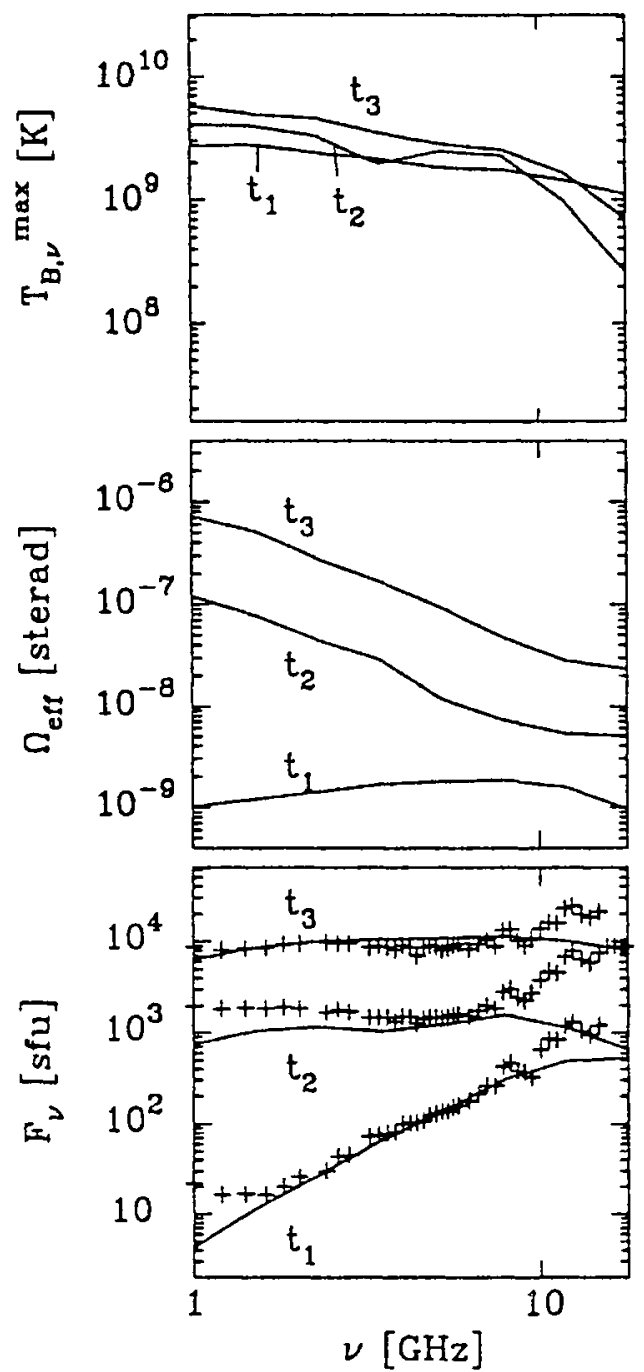

(b)

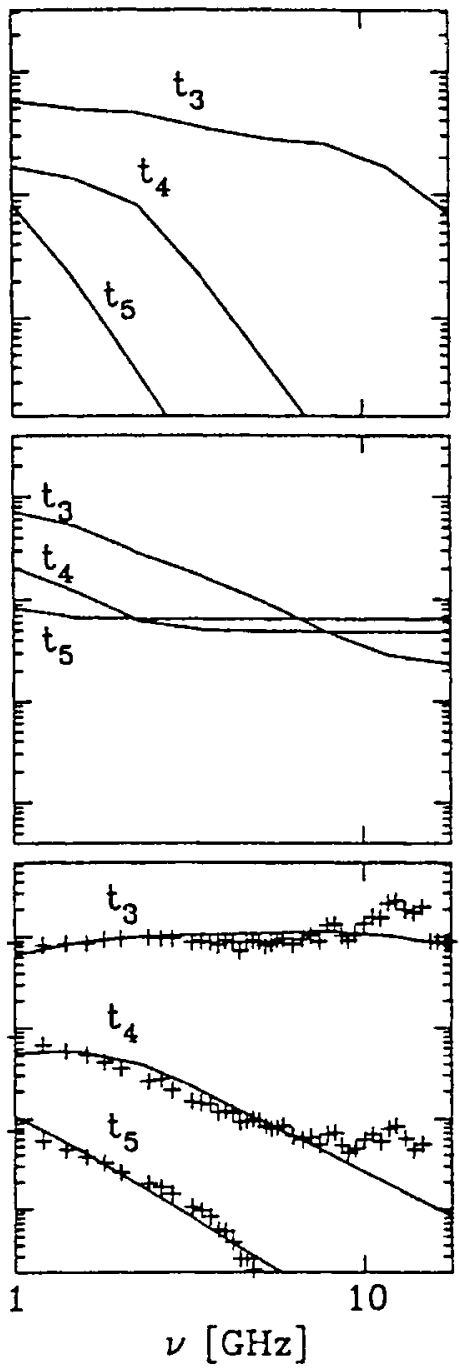

Fig. 6. A model fit to the observed spectral variation in the 1991 March 22 event at (a) rise phase and (b) decay phase. $t_{1}-t_{5}$ respectively refer to $22: 43: 20,22: 43: 50,22: 44: 10,22: 55: 00$, and 23:30:00 UT on 1991 March 22.

spectral variation in the rise phase is due to the change of loop size, $R(t)$, or the corresponding change of $\Omega_{\mathrm{eff}}(\nu)$ rather than that of $T_{B}(\nu)$. In this case, the delayed rise of lower frequency fluxes relative to high-frequency ones should be interpreted as due to the finite time taken for the structural change of the loop, which is about 1-2 min (cf., the time variations of $\Omega_{\mathrm{eff}}(\nu)$ and $T_{B}(\nu)$ in Figure 6(a)).

The maximum phase is reached when the loop is expanded to the size $\sim 300^{\prime \prime}$ as demanded to explain the observed flat and high-flux spectra as we worked out in Section 4.2 (curves denotes as $t_{3}$ in Figures 6(a) and 6(b)).

Figure 6(b) shows model-fits made to the decay-phase spectra. The spectral variation in this period is characterized by a gradual increase of the negative spectral slope of the spectra. A change of loop size cannot produce this effect because reducing loop size will lead to a positive spectral slope, and extending the 
loop size further seems too unrealistic. Required here would be a gradual decrease of average field strength in the source. We, therefore, consider the coronal trap model (see Section 3.2) with the assumption that a whole volume of nonthermal electrons is gradually shrinking to the loop top where fields are weakest. This, together with a softening of the electron power-law distribution $\left(\delta\left(t_{4}\right)=3.5\right.$ and $\delta\left(t_{5}\right)=4.0$ ), leads to a good fit to the spectra, with a negative spectral slope. The shrinking of the microwave source to the loop top lowers the turnover frequency of gyrosynchrotron spectra and the electron softening makes the spectral slope above the turnover frequency more steep. The former is conceivable in a case where the net survival time of high-energy electrons increases at a higher part of the loop due to decreasing Coulomb loss with height.

A common trend in the rise phase and the decay phase is that the effective source area $\Omega_{\mathrm{eff}}$, becomes more independent of frequency as we go away from the maximum phase (Figure 6). This means that the source in both the earlier rise and the later decay phase is smaller and more uniform. In contrast, the source at the maximum phase is highly inflated and the degree of magnetic field inhomogeneity is greatly enhanced, which leads to the increase of $\Omega_{\text {eff }}$ toward lower frequencies and thus the flux spectra become flat. The basic difference between the spectral evolution at the rise and that at the decay phase can be learned from Figure 6. The initial evolution of microwave spectra is mainly due to the change of $\Omega_{\mathrm{eff}}(\nu)$ associated with the loop dynamics, while the later evolution is mainly due to the change of $T_{B}(\nu)$ associated with the softening of nonthermal electrons.

Of many quantities involved with this model-fit, the most interesting one would be the time scale for the change of electron power-law index, $\delta$, in the particle evolution during the decay phase. We define a quantity, $\tau_{\delta}$, as the time taken for $\delta$ to increase by unity, and determine $\tau_{\delta}$ by comparing the microwave spectra calculated with $\delta$ and $\delta+1$ with the observed spectra. The results come out as $\tau_{\delta} \approx 3.3-46$ min depending on events (see the last column of Table II). A trend is not quite obvious with these four events alone except that $\tau_{\delta}$ is larger in a stronger event in general. It is, however, certain that $\tau_{\delta}$ is longer in the 1990 May 24 event than in the 1991 March 22 event, although the whole microwave activity is longer in the 1991 March 22 event. This might imply that the particle loss is involved with some internal dynamics which differs from event to event. According to our model scenario of these two big events, what is required is the preferential loss of higher-energy electrons over a time scale longer than $40 \mathrm{~min}$ in an inflated coronal trap where the fields are relatively weaker and the density of thermal particles is low. Coulomb loss may not be an adequate answer because the Coulomb loss is more efficient for lower-energy electrons and in denser medium. The radiative loss in the coronal trap would be too slow due to weaker field strength. The escape of high-energy electrons as a result of scattering by plasma turbulence could be a good candidate and the betatron loss (Bruggmann et al., 1994) could be another. 


\subsection{Correlations WITH X-RAYS}

In these events, microwaves at low frequencies are unusually extended in time and thus look similar to soft $\mathrm{X}$-rays, although a good correlation with hard $\mathrm{X}$-rays is still found at higher frequencies above $14 \mathrm{GHz}$. Such a time behavior might suggest a thermal origin for the low-frequency microwaves, in contradiction with our conclusion of the nonthermal origin for the microwaves based on the spectral fitting (Sections 4.2 and 4.3).

We have already mentioned in Section 2.2 that there are a few observational discrepancies that preclude this possibility. Another point gathered after modelanalysis is that the observed huge amount of radio flux cannot be produced by thermal gyrosynchrotron or by thermal free-free emission from soft X-ray particles with electron temperature at most $2 \times 10^{7} \mathrm{~K}$, although their emission measure is impressively high $\left(\int n_{e}^{2} \mathrm{~d} V \approx 10^{51} \mathrm{~cm}^{-3}\right)$.

Therefore, the question should shift from the thermal/nonthermal origin of the radiating particles to how the impulsive and extended components can coexist in an event and how such a frequency-dependence can result if both are of nonthermal origin. We believe that the key lies again in the influence of the field inhomogeneity upon the evolution of microwaves at different frequencies, along with the coronal trap's gradual shrinking to a weaker field region (i.e., the loop top). We thus propose, based on the model fit made in previous section, the following scenario:

(i) The whole microwave burst starts with an initial injection of nonthermal electrons at the loop top, which impulsively raises the fluxes at all frequencies. The duration of this injection should be no longer than that of high-frequency $(>14 \mathrm{GHz}$ ) microwaves and hard $\mathrm{X}$-rays which are as short as 1-2 min. Otherwise, longer microwave activity must have been seen at high frequencies as well as at the lower frequencies.

(ii) After the impulsive injection ceases, the evolution of microwaves is under the influence of the ambient medium which controls cooling efficiency of microwaveemitting electrons depending on the position within a loop. The electrons reaching footpoints will quickly die out due to the Coulomb loss efficient there. Since the Coulomb loss time is much shorter than the inferred injection time, the resulting radiation time profile is largely determined according to that of injection, which is over 1-2 min. This will be the case of high-frequency microwaves and perhaps the hard X-rays which occur in the stronger field region near footpoints. On the other hand, the lower frequency microwaves can persist until the flare activity in the coronal trap becomes entirely extinct, as they are emitted from the weaker field region near the loop top. The regular trend of longer microwave duration toward lower frequencies is then due to the gradual shrinking of the source region to the loop top.

The next concern would be whether the longer lifetime of low-frequency microwaves is entirely due to the cooling condition or partly due to continued acceleration, perhaps by interaction with plasma turbulence that builds up later. 
This important problem can properly be handled if a kinetic approach is used in combination with macroscopic dynamics of the loop (e.g., Bruggmann et al., 1994). This is beyond the scope of this paper in which the analysis is limited to spectral fitting at individual instances. However, the time scale, $\tau_{\delta}$, deduced from the present spectral fit may provide a piece of information useful in the detailed study of particle acceleration.

\section{Conclusions and Discussions}

In this paper we reported four microwave burst events which are characterized by flat and high flux spectra and a long duration (Section 2). We reviewed previous works on the flat spectra and those on the long-duration microwave activity to get a qualitative understanding of these events as a whole (Section 3). Based on these works, we attempted a quantitative analysis of the microwave spectra in terms of nonthermal gyrosynchrotron radiation from nonuniform, dipole magnetic loops (Section 4).

Before enumerating our conclusions, we wish to briefly discuss our use of the Dulk and Marsh (1982) expressions for nonthermal gyrosynchrotron emission from a single-power-law electron distribution. An often unstated assumption underlying these approximate expressions is that the single-power-law energy dependence for the electrons must hold true to very high energies $(>10 \mathrm{MeV})$. The presence of a high-energy cut-off in the electron energy distribution can, however, lead to decrease in radio emission at high frequencies (Ramaty, 1994, private communication). Often the electron distribution is assumed to extend to high energies with little justification since the commonly available information comes from hard X-ray spectrometers that give unreliable measurements above several hundred keV. For the 1990 May 24 flare, however, $\gamma$-ray observations are reported by Pelaez et al. (1994), and show that a single-power-law does fit the data over a wide energy range from $500 \mathrm{keV}$ to $10 \mathrm{MeV}$, justifying our use of the approximate expressions. A more explicit assumption that we must also justify in order to use the Dulk and Marsh (1982) expressions is that the important harmonics, $\nu / \nu_{B}$, lie in the range 10-100 over which the expressions are valid. In our continuous magnetic field model, there is always a range of gyrofrequencies involved with the emission at each frequency. As a way to judge the most effective harmonic at each frequency, we refer Figure 3(c) of Dulk and Marsh (1982), which plots the effective brightness temperature for the full expressions to find that our result, $2 \times 10^{9} \mathrm{~K} \leq T_{B, \nu}^{\max } \leq 6 \times 10^{9} \mathrm{~K}$ for $\nu=1-10 \mathrm{GHz}$ (Figure $4(\mathrm{~d})$ ), corresponds to $10<\nu / \nu_{B}^{\text {eff }}<100$ for a wide range of viewing angles. This justifies our use of Dulk and Marsh's (1982) simplified expressions to calculate spectra below $10 \mathrm{GHz}$. For higher frequencies, the lower harmonic emission from the strongest field region (i.e., the coronal base) might be involved which may make our calculation in this spectral regime less accurate. Our model is, however, mostly concerned with the lower frequency part of the spectrum. We also note that the lower frequency radiation coming from the loop 
top could involve high effective harmonics above a hundred. In this case, to use Dulk and Marsh's (1982) expression should mean that the microwave radiation is produced by ultrarelativistic electrons, of which presence is indicated by the $\gamma$-ray specturm of the 1990 May 24 flare (Pelaez et al., 1994).

Our results are summarized as follows: first, the flux spectra at the maximum phase appear as flat with fluxes ranging from $10^{2}$ to $10^{4}$ s.f.u. (Figure 1). As a first step to understanding this peculiar spectral behavior, we investigated how source inhomogeneity can lead to a flattening of gyrosynchrotron flux spectra in spite of increasing self-absorption at low frequencies. It is noted that the effective temperature of nonthermal particles can be made higher toward lower frequencies depending on source inhomogeneity. This property, together with increasing effective source area toward lower frequencies, tends to give flat flux spectra. Neither a thermal nor a uniform model share this radiative property and, therefore, they are excluded from the candidates (Figure 3). As specific results, we found that a flat microwave spectrum with $10^{2}-10^{4}$ s.f.u. may occur in a case where a magnetic loop is extended to an angular size of $\sim(0.7-7) \times 10^{-7}$ sterad and contains a huge amount $N(E>10 \mathrm{keV}) \approx 10^{36}-10^{38}$ of highly energetic particles with $\delta \approx 3-3.5$ distributed over the entire loop, spanning a wide range of magnetic fields from $1500 \mathrm{G}$ down to a few $\mathrm{G}$ (see Table II). These results based on spectral fitting are in support of previous works of other extended events based on the analysis of time profiles. As examples, the tendency for a harder electron population trapped in a weaker field region has been noted by Takakura, Uchida, and Kai (1968) and Kosugi, Dennis, and Kai (1988). The abundant number of relativistic electrons trapped in large magnetic loops has been noted in Vilmer, Kane, and Trottet (1982) and Kai, Kosugi, and Nitta (1985). Therefore, the nonuniform magnetic fields and abundant hard electrons trapped in the corona are perhaps the most fundamental factors for producing these large extended microwave bursts.

Second, a common pattern of spectral evolution is seen in these four events. We attempted to deduce the scenario underlying the spectral variation based on a model fit to instantaneous spectra by varying the loop size and power-law index of nonthermal electrons. In our results, the loop was initially of a smaller size and had a more uniform field distribution to emit radiation flux dominant at high frequency until it soon expanded to a big size to emit the flat, high-flux spectrum at the maximum phase. Such a loop expansion may alternatively be interpreted as the moving up of the microwave emitting region from a small loop to a larger loop in case of a multiple loop system (cf. Kocharev et al., 1994). The spectral change at the decay phase is reproduced by assuming confinement of the source region to a smaller one at the loop top and gradual softening of electron power-law. The former can happen in a case where the particle loss by Coulomb interaction decreases with height; the latter may be due to either escape of high-energy electrons or radiative energy loss. We note that the picture drawn here is in good agreement with sóme classical models of the type IV $\mu$ event (a long-lasting and broadband microwave emission) in general. Böhme et al. (1976) attempted to reproduce type IV $\mu$ spectra 
in terms of the 'core-halo model'. Our results qualitatively agree with their model in that we locate the short-lived high-frequency components at stronger fields near footpoints (core) and the long-lived low-frequency ones at weaker fields near loop-top (halo). Švestka (1976, p. 129) commented that the low-frequency radio fluxes may be due to magnetic field modulation of trapped particles while the high-frequency fluxes represent direct acceleration, referring to the type IV $\mu$ burst during the 1966 July 7 event (Enomé, 1969). Our model assumption of the timevarying coronal trap may provide a physical interpretation of the term 'magnetic modulation' that he used.

Third, our multi-frequency observation uncovers that these microwave bursts have both the impulsive and the extended components, which thus look similar to the hard X-rays and the soft X-rays, respectively. We showed that the coronal trap model can be made to fit this observation if properly combined with a nonuniform magnetic loop model. In this way, it is concluded that the gradual shrinking of the region of energetic electrons is responsible for the increasing microwave duration toward lower frequencies. However, it is yet to be checked whether the extended life is solely due to the cooling condition or partly requires additional injection or acceleration in a limited region of the magnetic loop. In the latter case, the detailed mechanism for subsequent acceleration (e.g., by shock waves) may deserve future study.

Finally, we wish to stress the distinction of these large extended events with the so-called 'gradual rise-and-fall' bursts. These two types of bursts sound similar in that both show long-duration activity and broadband spectra. The distinction can, however, be made in many ways. First of all, the gradual rise-and-fall components refer to weak events $(F(\nu>1 \mathrm{GHz})<40$ s.f.u. $)$ according to Švestka (1976, p. 140). The flux limit cited above is largely due to the presumed radiation mechanism which is the thermal free-free emission from soft X-ray particles (Shimabukuro, 1972; Hudson and Ohki, 1972). In contrast, extended burst seen at large flares accompany high fluxes $\left(F(\nu>1 \mathrm{GHz}) \geq 10^{2}\right.$ s.f.u. $)$ which is thus most likely due to nonthermal gyrosynchrotron emission as we worked out in this paper (Section 4.2). Another important difference is that the weak gradual bursts (or postbursts) show gradual rise while the large extended bursts like those presented here show impulsive rise although decay curves are extended. The gradual rise microwave flux as slow as soft X-rays implies that the weak gradual bursts are due to the thermal response of the ambient plasma to the heating by nonthermal particles. In contrast, the impulsive rise seen in large extended bursts will indicate that the microwave flux at all frequencies are directly from a nonthermal injection. Due to such a difference in underlying physics, the extended burst seen at large flares should not be regarded as just the high-energy version of weak gradual events. 


\section{Acknowledgements}

We thank the referee, Dr R. Ramaty, for helpful comments, particularly on the presence of ultrarelativistic electrons and on the use of simplified expressions for gyrosynchrotron radiation. We also thank Dr L. G. Kocharov for suggesting the possible two-loop system in the 1990 May 24 flare. We wish to thank Dr A. Krüger and Dr N. Vilmer for helpful discussions. We thank Dr C. Barat and Dr O. Terekhov for the information of Phebus data and Ms Kim Tolbert for providing the GOES data. Observation at OVRO was funded by NSF grants ATM-9013173 and AST8919770 to the California Institute of Technology. One of us (JWL) has been supported by NASA grant NAGW-3005 and NAGW-1972 during preparation of this paper.

\section{References}

Alissandrakis, C. E.: 1986, Solar Phys. 104, 207.

Alissandrakis, C. E. and Preka-Papadema, P.: 1984, Astron. Astrophys. 139, 507.

Böhme, A., Fürstenberg, F., Hildebrandt, J., Hoyng, P., Krüger, A., Saal, O., and Stevens, G. A.: 1976, HHI-STP-Report No. 6, Astronomical Institute Utrecht.

Böhme, A., Fürstenberg, F., Hildebrandt, J., Saal, O., Krüger, A., Hoyng, P., and Stevens, G. A.: 1977, Solar Phys. 53, 139.

Bruggmann, G., Vilmer, N., Klein, K.-L., and Kane, S. R.: 1994, Solar Phys. 149, 171.

Croom, D. L. and Powell, R. J.: 1971, Solar Phys. 20, 136.

Dennis, B. R.: 1988, Solar Phys. 118, 49.

Dennis, B. R. and Zarro, D. M.: 1993, Solar Phys. 146, 177.

Denton, R. E. and Feldman, U.: 1984, Astrophys. J. 286, 359.

Dulk, G. A. and Dennis, B. R.: 1982, Astrophys. J. 260, 875.

Dulk, G. A. and Marsh, K. A.: 1982, Astrophys. J. 259, 350.

Enomé, S.: 1969, Annals IQSY 3, 186.

Guidice, D. A. and Castelli, J. P.: 1975, Solar Phys. 44, 155.

Hachenberg, O. and Wallis, G.: 1961, Z. Astrophys. 52, 42.

Hudson, H. S. and Ohki, K.: 1972, Solar Phys. 23, 155.

Kai, K., Kosugi, T., and Nitta, N.: 1985, Publ. Astron. Soc. Japan 37, 105.

Klein, K.-L. and Trottet, G.: 1984, Astron. Astrophys. 141, 67.

Klein, K.-L., Trottet, G., and Magun, A.: 1986, Solar Phys. 104, 243.

Kocharov, L. G., Lee, J. W., Zirin, H., Kovaltsov, G. A., Usoskin, I. G., Pyle, K. R., Shea, M. A., and Smart, D. F.: 1994, Solar Phys., submitted.

Kosugi, T., Dennis, B. R., and Kai, K.: 1988, Astrophys. J. 324, 1118.

Kucera, T. A., Dulk, G. A., Kiplinger, A. L., Winglee, R. M., Bastian, T. S., and Graeter, M.: 1993, Astrophys. J. 412, 853.

Kucera, T. A., Dulk, G. A., Gary, D. E., M., and Bastian, T. S.: 1994, Astrophys. J., in press.

Neupert, W. M.: 1968, Astrophys. J. 153, L59.

Pelaez, F., Mandrou, P., Niel, M., Wena, B., Vilmer, N., Trottet, G., Lebrun, F., Paul, J., Terekhov, O., Sunyaev, R., Churazov, E., Gilfanov, M., Denisov, D., Kuznetsov, A., Dyachkov, A., and Khavenson, N.: 1992, Solar Phys. 140, 121.

Preka-Papadema, P. and Alissandrakis, C. E.: 1988, Astron. Astrophys. 191, 365.

Preka-Papadema, P. and Alissandrakis, C. E.: 1992, Astron. Astrophys. 257, 307.

Shimabukuro, F. I: 1992, Solar Phys. 23, 169.

Ramaty, R. and Petrosian, V.: 1972, Astrophys. J. 178, 169.

Stähli, M., Gary, D. E., and Hurford, G. J.: 1989, Solar Phys. 120, 351.

Stähli, M., Gary, D. E., and Hurford, G. J.: 1990, Solar Phys. 125, 343.

Švestka, Z.: 1976, Solar Flares, D. Reidel Publishing Co., Dordrecht, Holland.

Takakura, T.: 1972, Solar Phys. 26, 151. 
Takakura, T. and Scalise, E.: 1970, Solar Phys. 11, 434.

Takakura, T., Uchida, Y., and Kai, K.: 1968, Solar Phys. 4, 45.

Talon, R., Trottet, G., Vilmer, N., Barat, C., Dezalay, J.-P., Sunyaev, R., Terekhov, O., and Kuznetsov, A.: 1993, Solar Phys. 147, 137.

Tanaka, K.: 1969, Solar Terrestrial Activity Chart, January-June, 1969.

Tanaka, K. and Zirin, H.: 1985, Astrophys. J. 299, 1036.

Thomas, R. J., Starr, R., and Crannell, C. J.: 1985, Solar Phys. 95, 323.

Vilmer, N., Kane, S. R., and Trottet, G.: 1982, Astron. Astrophys. 108, 306. 